\title{
Liver enzyme elevation in patients with ankylosing spondylitis treated with tumor necrosis factor inhibitors: a single-center historical cohort study
}

\author{
Su Jin Choi ${ }^{1}$, Ji Seon $\mathrm{Oh}^{2}$, Seokchan Hong ${ }^{1}$, Chang-Keun Lee ${ }^{1}$, Bin Yoo ${ }^{1}$, and Yong-Gil Kim
}

${ }^{1}$ Division of Rheumatology, Department of Internal Medicine and ${ }^{2}$ Clinical Research Center, Asan Medical Center, University of Ulsan College of Medicine, Seoul, Korea

Received: November 14, 2018 Revised : February 20, 2019 Accepted: February 27, 2019

\section{Correspondence to}

Yong-Gil Kim, M.D.

Division of Rheumatology,

Department of Internal

Medicine, Asan Medical Center, University of Ulsan College of Medicine, 88 Olympic-ro 43-gil, Songpa-gu, Seoul 05505, Korea

Tel: $+82-2-3010-3279$

Fax: +82-2-3010-6969

E-mail: bestmd200o@amc.seoul.kr
Background/Aims: Tumor necrosis factor inhibitors (TNFi) have been known to induce liver enzyme elevation, sometimes associated with viral reactivation or toxic hepatitis. We evaluated the incidence and risk factors of TNFi-associated liver enzyme elevation in Korean ankylosing spondylitis (AS) patients who previously had normal liver enzymes.

Methods: Retrospectively, we collected data from the records of 363 AS patients treated with TNFi at a tertiary hospital from 2003 to 2017 . Liver enzyme elevation was defined as abnormal elevation of aspartate aminotransferase and/or alanine aminotransferase levels on two or more consecutive visits. Patients with previously diagnosed liver disease were excluded.

Results: The incidence of liver enzyme elevation was $23.7 \%$ (occurring in 86 of 363 patients). The median duration of TNFi exposure before liver enzyme elevation was 3.72 months (interquartile range, 1.77 to 12.51 ). There was no difference in the occurrence of liver enzyme elevation with concomitant disease-modifying anti-rheumatic drugs and TNFi compared to TNFi alone (23.9\% vs. $23.6 \%$ ). In multivariate analysis, the hazard ratios for liver enzyme elevation were 4.62 (95\% confidence interval [CI], 1.43 to 15.01) for male sex, 4.06 (95\% CI, 2.11 to 7.84 ) for underlying non-alcoholic fatty liver disease, and 2.53 (95\% CI, 1.38 to 4.64 ) for hyperlipidemia. After switching to another TNFi, the liver enzyme elevation was not normalized in nine of 13 patients.

Conclusions: Liver enzyme elevation was observed in a quarter of patients with AS receiving a TNFi. Male sex, non-alcoholic fatty liver disease, and hyperlipidemia were independent risk factors for liver enzyme elevation. Switching to another TNFi had a limited effect on restoring normal liver enzyme levels.

Keywords: Aspartate aminotransferases; Alanine transaminase; Spondylitis, ankylosing; Tumor necrosis factor-alpha

\section{INTRODUCTION}

Ankylosing spondylitis (AS) is a chronic inflammatory rheumatic disease affecting sacroiliac joints, the axi- al skeleton, peripheral joints, and entheses. Although conventional treatment including non-steroidal anti-inflammatory drugs (NSAIDs) and physical therapy is the basis of AS therapy, it has limited efficacy [1]. Tu- 
Table 1. Comparison of demographic, clinical, and laboratory findings in AS patients receiving TNF inhibitors

\begin{tabular}{|c|c|c|c|}
\hline Characteristic & Normal liver enzyme $(n=277)$ & Elevated liver enzyme $(n=86)$ & $p$ value \\
\hline Age at diagnosis, yr & $33.9(14.2)$ & $33.5(13.6)$ & 0.852 \\
\hline Age at onset of TNF inhibitor, yr & $37.9(13.7)$ & $37.1(13.1)$ & 0.628 \\
\hline Disease duration, mon & $110.19(78.4)$ & $121.90(71.1)$ & 0.218 \\
\hline Male sex & $198(71.5)$ & $78(90.7)$ & $<0.001$ \\
\hline Diabetes mellitus & $7(2.5)$ & $7(8.1)$ & 0.026 \\
\hline Hypertension & $48(17 \cdot 3)$ & $19(22.1)$ & 0.320 \\
\hline Chronic kidney disease & $7(2.5)$ & o & 0.205 \\
\hline Hyperlipidemia & $72(26.0)$ & $48(55.8)$ & $<0.001$ \\
\hline NAFLD & $12(4 \cdot 3)$ & $19(22.1)$ & $<0.001$ \\
\hline Smoker & $137 / 270(50.7)$ & $51 / 85(60.0)$ & 0.136 \\
\hline Hazardous drinking & $16 / 175(9.1)$ & $5 / 48(10.4)$ & 0.783 \\
\hline $\mathrm{BMI}, \mathrm{kg} / \mathrm{m}^{2}$ & $24.08(6.25)$ & $25.53(2.94)$ & 0.044 \\
\hline ANA & $15 / 123(12.2)$ & $3 / 41(7 \cdot 3)$ & 0.566 \\
\hline HLA-B27 & $228 / 250(91.2)$ & $71 / 77(92.2)$ & 0.782 \\
\hline History of AST/ALT elevation & $53(19.1)$ & $22(25 \cdot 6)$ & 0.197 \\
\hline \multicolumn{4}{|l|}{ Concomitant medication } \\
\hline NSAID & $254(91.7)$ & $80(93.0)$ & 0.692 \\
\hline Statin & $36(13.0)$ & $10(11.6)$ & 0.739 \\
\hline Latent TB prophylaxis & $106(38.3)$ & $39(45 \cdot 3)$ & 0.241 \\
\hline Corticosteroid & $72(26.0)$ & $16(18.6)$ & 0.163 \\
\hline DMARDs & $83(30.0)$ & $26(30.2)$ & 0.805 \\
\hline \multicolumn{4}{|l|}{ TNF inhibitor } \\
\hline Adalimumab & $117(42.2)$ & $43(50.0)$ & 0.205 \\
\hline Etanercept & $81(29.2)$ & $27(31.4)$ & 0.703 \\
\hline Infliximab & $56(20.2)$ & $8(9 \cdot 3)$ & 0.020 \\
\hline Golimumab & $23(8.3)$ & $8(9 \cdot 3)$ & 0.825 \\
\hline \multicolumn{4}{|l|}{ Laboratory findings } \\
\hline Baseline creatinine, mg/dL & $0.78 \pm 0.17$ & $0.82 \pm 0.15$ & 0.025 \\
\hline Baseline AST, IU/L & $20.33 \pm 5.36$ & $21.60 \pm 6.07$ & 0.083 \\
\hline Baseline ALT, IU/L & $13.65 \pm 6.17$ & $17.53 \pm 6.82$ & $<0.001$ \\
\hline Baseline ALP, IU/L & $82.82 \pm 26.32$ & $86.53 \pm 20.64$ & 0.236 \\
\hline Baseline bilirubin, mg/dL & $0.61 \pm 0.23$ & $0.67 \pm 0.22$ & 0.048 \\
\hline Baseline albumin, mg/dL & $4.18 \pm 2.37$ & $4.15 \pm 0.33$ & 0.908 \\
\hline Baseline ESR, mm/hr & $43.63 \pm 28.13$ & $46.57 \pm 25.78$ & 0.389 \\
\hline Baseline CRP, mg/dL & $1.92 \pm 2.25$ & $2.43 \pm 2.38$ & 0.066 \\
\hline
\end{tabular}

Values are presented as mean $\pm \mathrm{SD}$ or number (\%).

AS, ankylosing spondylitis; TNF, tumor necrosis factor; NAFLD, non-alcoholic fatty liver disease; BMI, body mass index; ANA, antinuclear antibody; HLA-B27, human leukocyte antigen-B27; AST, aspartate aminotransferase; ALT, alanine aminotransferase; NSAID, non-steroidal anti-inflammatory drug; TB, tuberculosis; DMARD, disease-modifying anti-rheumatic drug; ALP, alkaline phosphatase; ESR, erythrocyte sedimentation rate; CRP, C-reactive protein. 
mor necrosis factor inhibitor (TNFi), which is used to treat rheumatoid arthritis (RA) and inflammatory bowel disease (IBD), shows efficacy for treating AS with poor response to conventional treatments. However, unlike the options for patients with RA and IBD, the treatment options for AS patients, other than TNFi, are limited.

There are major concerns regarding the adverse effects of TNFi, such as tuberculosis, malignancy, and infection [2]. Hepatotoxicity is also a relatively common adverse effect associated with the use of TNFi [3]. In a United States Food and Drug Administration post-marketing surveillance program, treatment with infliximab or etanercept was associated with more than 130 cases of hepatotoxicity within 5 years [4]. The severity of hepatotoxicity ranges from mild liver enzyme elevation to acute liver failure, though liver failure is very rare $[5,6]$. The exact mechanism of liver enzyme elevation is unclear, although some reports showed that TNFi was associated with autoimmunity, non-alcoholic fatty liver disease (NAFLD), and reactivation of viral hepatitis [7-10].

The hepatotoxicity of TNFi in autoimmune diseases including RA, psoriatic arthritis, and IBD has been reported in several studies $[7,11,12]$. However, there has been little evidence of liver enzyme elevation related to TNFi in AS patients $[8,13]$. The influence of concomitant hepatotoxic medications such as disease-modifying anti-rheumatic drugs (DMARDs) and immunosuppressants may be less in AS than in other autoimmune diseases. Furthermore, different environmental and genetic factors may influence susceptibility to adverse effects. Therefore, we investigated the incidence and risk factors of liver enzyme elevation related to TNFi in Korean AS patients who previously had normal liver enzymes.

\section{METHODS}

\section{Study population}

We retrospectively reviewed the medical records of 363 AS patients receiving TNFi in a tertiary hospital from January 2003 to December 2017. The diagnosis of AS was confirmed according to the modified New York criteria [14]. All patients had normal serum liver enzyme levels at least 6 months before initiation of TNFi treatment. Patients with a history of chronic viral hepatitis, other drug-induced hepatitis, or alcoholic liver disease were excluded. Adalimumab, etanercept, infliximab, or golimumab were used to treat AS patients.

The following demographic and clinical information was collected: demographic data including age, sex, body mass index (BMI, $\mathrm{kg} / \mathrm{m}^{2}$ ), and hazardous drinking according to World Health Organization criteria [15]; comorbidities such as diabetes mellitus, hypertension, hyperlipidemia, and NAFLD; concomitant medication including NSAIDs, DMARDs (methotrexate and sulfasalazine), anti-tuberculosis drugs, corticosteroids, and statins; laboratory data including aspartate aminotransferase (AST), alanine aminotransferase (ALT), alkaline phosphatase, bilirubin, antinuclear antibody (ANA), human leukocyte antigen-B27 (HLA-B27) erythrocyte sedimentation rate (ESR), and C-reactive protein (CRP).

Liver enzyme elevation was defined as serum AST and/or ALT levels greater than the upper limit of normal (ULN) and consecutively elevated for at least two visits. NAFLD was identified by imaging including ultrasound and computed tomography, which were performed when clinically indicated. The study was approved by the Institutional Review Board (2017-1313) of the Asan Medical Center at Seoul, Korea. Informed consent was waived due to a retrospective design of our study.

\section{Statistical analysis}

Continuous variables were expressed as mean \pm standard deviation or median (interquartile range [IQR]). They were compared using the Student's $t$ test or the Mann-Whitney U test. Categorized data were expressed as percentiles and compared by chi-square or Fisher's exact test. Cox regression analysis was performed to assess hazard ratio (HR) and 95\% confidence interval (CI) for liver enzyme elevation. All data were analyzed using SPSS version 24.0 software (IBM Co., Armonk, NY, USA). A $p<0.05$ was considered statistically significant.

\section{RESULTS}

\section{Characteristics in TNFi users with or without liver enzyme elevation}

The demographic and clinical characteristics of AS patients are shown in Table 1. Among 363 AS patients treated with TNFi, liver enzyme elevation was observed in 


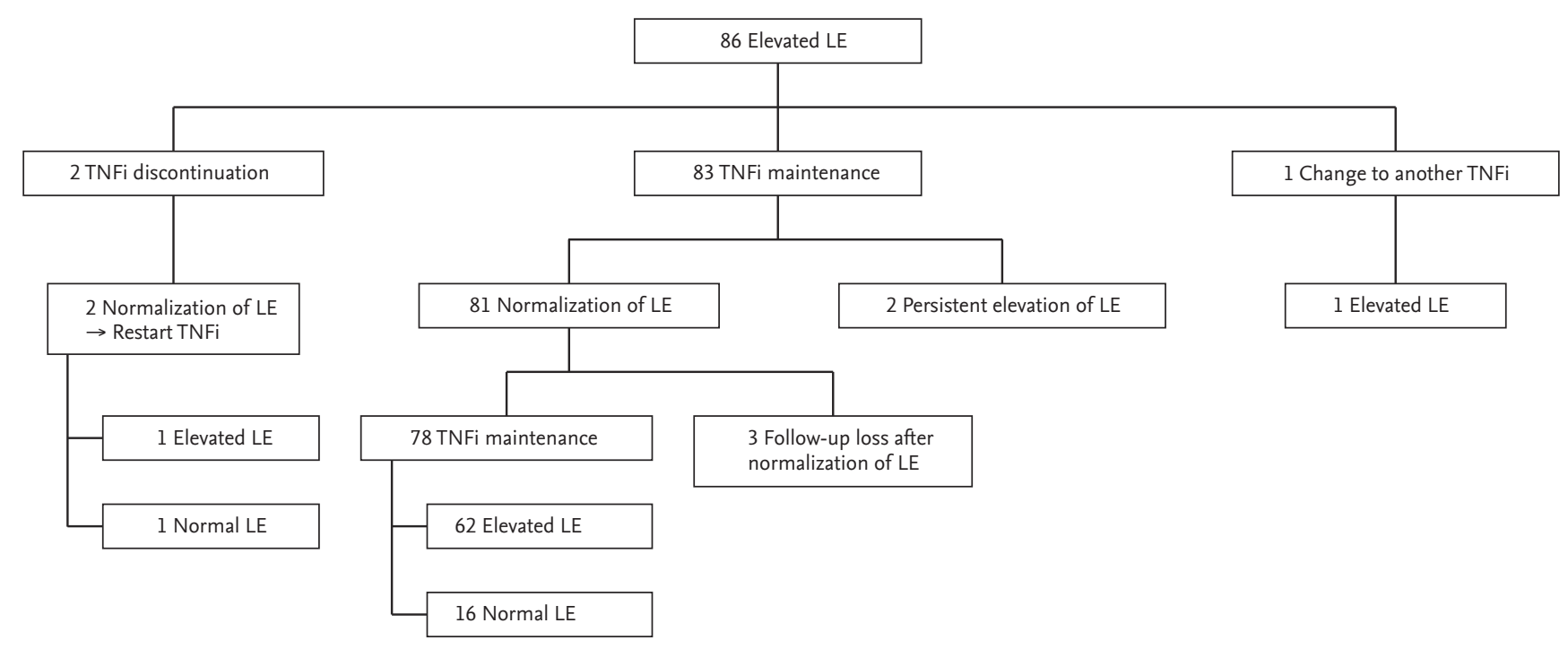

Figure 1. Outcomes of liver enzyme (LE) elevation in patients treated with tumor necrosis factor inhibitor (TNFi).

86 patients (23.7\%). Patients with elevated liver enzyme showed a significantly higher prevalence of male sex, diabetes mellitus, hyperlipidemia, and NAFLD compared to patients with normal liver enzymes. Hyperlipidemia was diagnosed after treatment with TNFi in 32 patients (37.2\%) in the elevated liver enzyme group and 52 patients $(18.8 \%)$ in the normal liver enzyme group. NAFLD was diagnosed after starting TNFi treatment in 16 patients (18.6\%) in the elevated liver enzyme group and seven patients $(2.5 \%)$ in the normal liver enzyme group. There was no significant difference in the proportion of hazardous alcohol use between two groups (10.4\% in elevated liver enzyme group vs. 9.1\% in normal liver enzyme group). Normal liver enzyme group included patients with inflammatory bowel diseases (four patients), coronary artery diseases (three patients), malignancies (two patients), and polycythemia vera (one patient). Creatinine, ALT, and bilirubin at baseline were relatively increased in the elevated liver enzyme groups; however, the use of other medication, including NSAIDs, statins, and DMARDs, were not different between the two groups. In particular, the use of concomitant DMARDs was observed in 30.0\% (methotrexate 60 patients, sulfasalazine 36 patients) of patients in the normal liver enzyme group and $30.2 \%$ (methotrexate 19 patients, sulfasalazine 10 patients) of patients in the elevated liver enzyme group. The incidence of liver enzyme elevation in patients who
Table 2. Level of elevated AST/ALT in AS patients receiving TNF inhibitors

\begin{tabular}{lc}
\hline Liver enzyme levels & Elevated liver enzyme $(\mathrm{n}=86)$ \\
\hline $2 \geq$ AST or ALT $>1 \times \mathrm{ULN}$ & $54(62.8)$ \\
$3 \geq$ AST or ALT $>2 \times \mathrm{ULN}$ & $19(22.1)$ \\
$5 \geq$ AST or ALT $>3 \times \mathrm{ULN}$ & $9(10.5)$ \\
AST or ALT $>5 \times \mathrm{ULN}$ & $4(4.6)$ \\
\hline
\end{tabular}

Values are presented as number (\%).

AST, aspartate aminotransferase; ALT, alanine aminotransferase; AS, ankylosing spondylitis; TNF, tumor necrosis factor; ULN, upper limit of normal.

received concomitant DMARDs and TNFi treatment was similar to that in patients who received TNFi alone (23.9\% vs. $23.6 \%, p=0.962)$.

The most frequently prescribed TNFi was adalimum$\mathrm{ab}$, which was used in $42.2 \%$ of the normal liver enzyme group and in $50.0 \%$ of the elevated liver enzyme group. The proportion of patients receiving infliximab was higher in the normal liver enzyme group than in the elevated liver enzyme group (20.2\% vs. 9.3\%, $p=0.020$ ).

In the elevated liver enzyme group, the median time from TNFi initiation to onset of liver enzyme elevation (latency) was 3.72 months (IQR, 1.77 to 12.51) and the mean peak level of AST/ALT was 60.6/83.9 IU/L. Of the 86 patients in the elevated liver enzyme group, 37.2\% 
Table 3. Risk factors of liver enzyme elevation in AS patients receiving TNF inhibitors

\begin{tabular}{|c|c|c|c|c|}
\hline Characteristic & Univariate analysis & $p$ value & Multivariate analysis & $p$ value \\
\hline Male sex & $3.23(1.56-6.70)$ & 0.002 & $4.62(1.43-15.01)$ & 0.011 \\
\hline Diabetes mellitus & $2.40(1.11-5.20)$ & 0.026 & & \\
\hline Hypertension & $1.26(0.76-2.10)$ & 0.375 & & \\
\hline Hyperlipidemia & $2.82(1.84-4.32)$ & $<0.001$ & $2.53(1.38-4.64)$ & 0.003 \\
\hline NAFLD & $3.90(2.33-6.51)$ & $<0.001$ & $4.06(2.11-7.84)$ & $<0.001$ \\
\hline Smoker & $1.34(0.87-2.08)$ & 0.182 & & \\
\hline Hazardous drinking & $1.23(0.49-3.12)$ & 0.658 & & \\
\hline BMI & $1.03(1.01-1.06)$ & 0.010 & & \\
\hline Baseline creatinine & $3.25(0.98-10.75)$ & 0.054 & & \\
\hline Baseline ALT & $1.06(1.04-1.08)$ & $<0.001$ & $1.14(1.09-1.19)$ & $<0.001$ \\
\hline Baseline bilirubin & $2.10(0.86-5.15)$ & 0.104 & & \\
\hline Baseline CRP & $1.08(1.00-1.17)$ & 0.570 & & \\
\hline ANA & $0.63(0.20-2.07)$ & 0.453 & & \\
\hline \multicolumn{5}{|l|}{ Concomitant medication } \\
\hline NSAID & $0.96(0.42-2.20)$ & 0.918 & & \\
\hline Statin & $0.72(0.37-1.39)$ & 0.322 & & \\
\hline Latent TB prophylaxis & $1.20(0.78-1.83)$ & 0.401 & & \\
\hline Corticosteroid & $0.66(0.38-1.13)$ & 0.130 & & \\
\hline DMARDs & $1.08(0.71-1.65)$ & 0.732 & & \\
\hline \multicolumn{5}{|l|}{ TNF inhibitor } \\
\hline Adalimumab & $1.47(0.96-2.24)$ & 0.078 & & \\
\hline Etanercept & $1.01(0.64-1.60)$ & 0.961 & & \\
\hline Infliximab & $0.42(0.20-0.88)$ & 0.020 & & \\
\hline Golimumab & $1.08(0.52-2.23)$ & 0.843 & & \\
\hline
\end{tabular}

Values are presented as hazard ratios (95\% confidence interval). Variables with $p<0.2$ on univariate analysis were included in multivariate analysis and variables with $p<0.05$ were presented.

AS, ankylosing spondylitis; TNF, tumor necrosis factor; NAFLD, non-alcoholic fatty liver disease; BMI, body mass index; ALT, alanine aminotransferase; CRP, C-reactive protein; ANA, antinuclear antibody; NSAID, non-steroidal anti-inflammatory drug; TB, tuberculosis; DMARD, disease-modifying anti-rheumatic drug.

(32/86) developed AST and/or ALT elevation greater than two times the ULN. The incidence of AST and/or ALT elevation greater than five times the ULN was $4.6 \%$ (4/86) (Table 2). The four patients with liver enzyme elevation greater than five times the ULN were all males and had either NAFLD (two patients) or hyperlipidemia (three patients) or both. Furthermore, among the four patients, three underwent ANA testing and the results were all negative. However, the peak total bilirubin level in the elevated AST/ALT group was $1.3 \mathrm{mg} / \mathrm{dL}$ (reference range $\leq 1.2 \mathrm{mg} / \mathrm{dL}$ ).

\section{Clinical factors associated with liver enzyme eleva- tion in patients receiving TNFi}

Table 3 shows the risk factors for liver enzyme elevation in TNFi users. Univariate analysis showed that male sex, diabetes mellitus, hyperlipidemia, NAFLD, and high BMI were associated with liver enzyme elevation. Infliximab was weakly associated with liver enzyme elevation compared to other TNFi (HR, 0.42; 95\% CI, 0.20 to o.88). In multivariate analysis, the independent risk factors of liver enzyme elevation were male sex (HR, 4.62; 95\% CI, 1.43 to 15.01 ), NAFLD (HR, 4.06; 95\% CI, 2.11 to 7.84 ), and hyperlipidemia (HR, 2.53; 95\% CI, 1.38 to 4.64 ). The use of 
Table 4. Switching to another TNF inhibitor in AS patients with liver enzyme elevation

\begin{tabular}{lcc}
\hline Switching TNF inhibitor & $\begin{array}{c}\text { Switching to } \\
\text { another TNF } \\
\text { inhibitor } \\
(\mathrm{n}=13)\end{array}$ & $\begin{array}{c}\text { Normaliza- } \\
\text { tion after } \\
\text { switching } \\
(\mathrm{n}=4)\end{array}$ \\
\hline Adalimumab $\rightarrow$ Etanercept & 5 & 2 \\
\hline Adalimumab $\rightarrow$ Infliximab & 2 & 0 \\
Etanercept $\rightarrow$ Adalimumab & 2 & 0 \\
Etanercept $\rightarrow$ Golimumab & 1 & 0 \\
\hline Infliximab $\rightarrow$ Golimumab & 1 & 0 \\
Golimumab $\rightarrow$ Adalimumab & 2 & 2 \\
\hline
\end{tabular}

TNF, tumor necrosis factor; AS, ankylosing spondylitis.

concomitant medications and laboratory findings such as ANA, HLA-B27, baseline ESR, and CRP were not associated with liver enzyme elevation.

\section{Effects of maintaining or discontinuing TNFi treatment in patients with liver enzyme elevation}

During the follow-up period, most patients $(83$ out of 86 patients) with elevated liver enzyme were maintained in TNFi treatment, and 81 of those patients showed decreases in liver enzyme within normal range. Among 78 patients who maintained TNFi after liver enzyme normalization, 62 patients experienced re-elevation of liver enzyme. Two patients showed improvements in liver enzyme after discontinuation of TNFi, which was re-elevated after resuming TNFi (Fig. 1).

Among the 86 patients with liver enzyme elevation, 13 patients were switched to another TNFi during the follow-up period. Liver enzyme elevation even after switching to another TNFi was observed in nine of 13 patients; however, four patients developed normal liver enzymes after switching (two patients switched from adalimum$\mathrm{ab}$ to etanercept and two patients switched from golimumab to adalimumab) (Table 4).

\section{DISCUSSION}

In this study, we determined that liver enzyme elevation associated with TNFi therapy is relatively common in Korean AS patients. Male sex, NAFLD, and hyperlipidemia were independently associated with liver enzyme

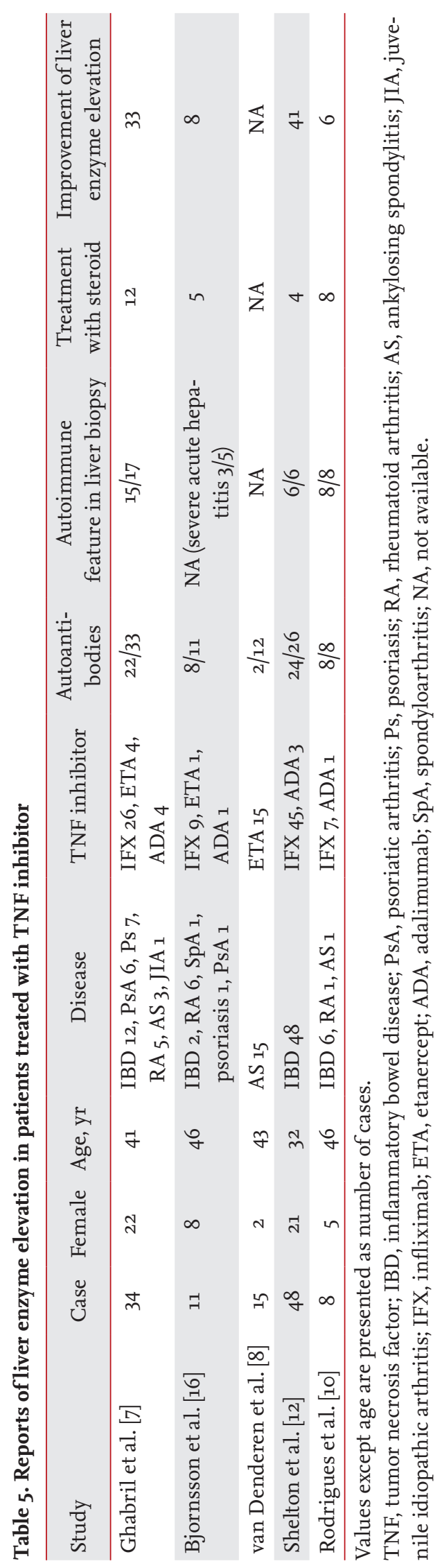


elevation. Switching to another TNFi did not always restore normal serum liver enzyme levels.

The incidence of liver enzyme elevation was $23.7 \%$ in our AS patients treated with TNFi. Previous studies showed various incidences, perhaps due to different definitions of liver enzyme elevation $[8,16,17]$. If the definition of liver enzyme elevation is confined to more than two times the ULN, the incidence of elevated AST/ ALT is $8.8 \%$ (occurring in 32 of 363 TNFi recipients) in our study. This result is comparable to those reported previously $[8,12]$. Furthermore, the median latency was 3.72 months (almost 15 weeks), which is also similar to the results of previous studies showing 14 to 18 weeks of median latency for TNFi-induced hepatotoxicity $[12,16]$.

There are several hypotheses regarding the mechanism of TNFi-related hepatotoxicity in previous studies (Table 5). One hypothesis posits autoimmunity as the cause. It is well known that some individuals receiving TNFi therapy develop autoimmunity with high levels of autoantibodies. In a previous study, positive ANA, a marker of autoimmune hepatitis, was found at the time of diagnosis of drug-induced liver injury in $73 \%$ of patients (8/11) receiving TNFi [16]. However, a positive ANA was not found in our AS patients who developed liver enzyme elevation greater than five times the ULN.

On the other hand, liver steatosis has also been considered a cause of liver enzyme elevation. Underlying NAFLD is a known risk factor of drug-induced liver disease [18]. In a previous study of AS patients treated with etanercept, high BMI was associated with liver enzyme elevation, and hepatic steatosis was observed in five of six patients with liver enzyme elevation [8]. These findings suggest that, although the mechanism has not been fully identified $[19,20]$, aggravation of NAFLD might have contributed substantially to the development of liver enzyme elevation in our patients. Recent studies have yielded interesting clues about the association between TNFi and NAFLD in IBD patients [21]. Intrahepatic fat disposition was inhibited by TNFi in individuals with active IBD, while in those with inactive IBD, a metabolic mechanism that induced fatty liver disease was promoted by TNFi, associated with insulin resistance and weight gain [22]. Therefore, liver enzyme elevation in our AS patients after several months of TNFi exposure might have resulted from fatty liver change through a metabolic mechanism followed by inflammation con- trol.

In the present study, male sex was the other risk factor of liver enzyme elevation in patients treated with TNFi. In drug-induced liver injury, women are usually more susceptible [23]. However, some medications, such as azathioprine, are more likely to cause liver enzyme elevation in men than in women [24]. Pharmacokinetics and pharmacodynamics differ in men and women due to such factors as hormonal effects, body weight, body composition, and gender-specific differences in the immune system [25]. AS also shows a male predominance. Specific genetic polymorphism in AS might contribute to the gender difference in liver enzyme elevation.

Infliximab showed a weaker association with liver enzyme elevation than other TNFi in univariate analysis, but the association disappeared in the multivariate analysis. This finding was different from those of previous reports showing that infliximab was the most common TNFi to induce liver injury [16]. The reason for this result is not clear; however, infliximab is known to induce more ANA reactivity than etanercept and to promote liver enzyme elevation with autoimmunity $[16,26]$. In contrast, in a previous study involving individuals with AS, the mean levels of serum liver enzyme at 12 months after initiation of TNFi were higher with adalimumab than infliximab or etanercept treatment, although they were within the normal range [13]. The dose of infliximab also might be one of the causes of this inconsistency. In previous studies, patients treated with a lower dose of infliximab developed liver enzyme elevation more frequently than patients treated with a standard dose $[12,16]$. The standard dose of infliximab is generally higher in patients with $\mathrm{AS}(5 \mathrm{mg} / \mathrm{kg})$ than that in those with RA (3 mg/kg) [27]. Therefore, the results in our AS patients suggest that the use of infliximab is not a risk factor for liver enzyme elevation and that infliximab is comparable to other TNFi regarding hepatotoxicity.

Cross-reactivity when switching to another TNFi occurred uncommonly in previous studies [28-30]. This finding might be due to the differences of drug chemical structure and polymorphism of the TNF-alpha gene [31]. However, we showed that other TNFi also induced liver enzyme elevation in some patients. This observation may relate to the similar mechanisms of effect among tumor necrosis factor inhibitors, and to the possible function of TNF to promote liver regeneration [32]. 
This study had several limitations. Many other medical conditions that could affect liver enzyme elevation were not fully identified in this retrospective study. Also, liver biopsies were not examined, so the mechanism of hepatotoxicity was not determined.

In conclusion, we observed liver enzyme elevation after TNFi exposure in $23.7 \%$ of AS patients who previously had normal liver enzyme levels. The risk of liver enzyme elevation was increased in male AS patients and in AS patients with NAFLD or hyperlipidemia. Switching to another TNFi appears to have limited utility for restoring normal liver function.

\section{KEY MESSAGE}

1. Liver enzyme elevation was a relatively common adverse effect of tumor necrosis factor (TNF) inhibitor therapy in patients with ankylosing spondylitis.

2. Male sex, non-alcoholic fatty liver disease, and hyperlipidemia were important factors associated with liver enzyme elevation in patients with ankylosing spondylitis treated with TNF inhibitors.

3. Liver enzyme elevation was often observed even after switching to another TNF inhibitor.

\section{Conflict of interest}

No potential conflict of interest relevant to this article was reported.

\section{Acknowledgments}

This work was supported by the Asan Institute for Life Sciences (grant number 2017-655).

\section{REFERENCES}

1. van der Heijde D, Ramiro S, Landewe R, et al. 2016 Update of the ASAS-EULAR management recommendations for axial spondyloarthritis. Ann Rheum Dis 2017;76:978991.

2. Scheinfeld N. A comprehensive review and evaluation of the side effects of the tumor necrosis factor alpha blockers etanercept, infliximab and adalimumab. J Dermatolog
Treat 2004;15:280-294.

3. van der Heijde D, Kivitz A, Schiff MH, et al. Efficacy and safety of adalimumab in patients with ankylosing spondylitis: results of a multicenter, randomized, double-blind, placebo-controlled trial. Arthritis Rheum 2006;54:2136-2146.

4. Food and Drug Administration. Briefing Document. Safety alerts for drugs, biologics medical devices, and dietary supplements. Remicade (Infliximab) [Internet]. Silver Spring (MD): Food and Drug Administration, c2004 [cited 2019 Sep 30]. Available from: https://www.accessdata.fda.gov/scripts/cder/daf/index.cfm?event=overview. process\&ApplNo=103772.

5. Parekh R, Kaur N. Liver injury secondary to anti-TNF-alpha therapy in inflammatory bowel disease: a case series and review of the literature. Case Rep Gastrointest Med 2014;2014:956463.

6. Tobon GJ, Canas C, Jaller JJ, Restrepo JC, Anaya JM. Serious liver disease induced by infliximab. Clin Rheumatol 2007;26:578-581.

7. Ghabril M, Bonkovsky HL, Kum C, et al. Liver injury from tumor necrosis factor- $\alpha$ antagonists: analysis of thirty-four cases. Clin Gastroenterol Hepatol 2013;11:558564.

8. van Denderen JC, Blom GJ, van der Horst-Bruinsma IE, Dijkmans BA, Nurmohamed MT. Elevated liver enzymes in patients with ankylosing spondylitis treated with etanercept. Clin Rheumatol 2012;31:1677-1682.

9. Cansu DU, Kalifoglu T, Korkmaz C. Short-term course of chronic hepatitis B and C under treatment with etanercept associated with different disease modifying antirheumatic drugs without antiviral prophylaxis. J Rheumatol 2008;35:421-424.

10. Rodrigues S, Lopes S, Magro F, et al. Autoimmune hepatitis and anti-tumor necrosis factor alpha therapy: a single center report of 8 cases. World J Gastroenterol 2015;21:7584-7588.

11. Sokolove J, Strand V, Greenberg JD, et al. Risk of elevated liver enzymes associated with TNF inhibitor utilisation in patients with rheumatoid arthritis. Ann Rheum Dis 2010;69:1612-1617.

12. Shelton E, Chaudrey K, Sauk J, et al. New onset idiosyncratic liver enzyme elevations with biological therapy in inflammatory bowel disease. Aliment Pharmacol Ther 2015;41:972-979.

13. Capkin E, Karkucak M, Cosar AM, et al. Treatment of an- 
kylosing spondylitis with TNF inhibitors does not have adverse effect on results of liver function tests: a longitudinal study. Int J Rheum Dis 2015;18:548-552.

14. Goie The HS, Steven MM, van der Linden SM, Cats A. Evaluation of diagnostic criteria for ankylosing spondylitis: a comparison of the Rome, New York and modified New York criteria in patients with a positive clinical history screening test for ankylosing spondylitis. Br J Rheumatol 1985;24:242-249.

15. Korean Association for the Study of the Liver (KASL). KASL clinical practice guidelines: management of alcoholic liver disease. Clin Mol Hepatol 2013;19:216-254.

16. Bjornsson ES, Gunnarsson BI, Grondal G, et al. Risk of drug-induced liver injury from tumor necrosis factor antagonists. Clin Gastroenterol Hepatol 2015;13:602-608.

17. Maini R, St Clair EW, Breedveld F, et al. Infliximab (chimeric anti-tumour necrosis factor alpha monoclonal antibody) versus placebo in rheumatoid arthritis patients receiving concomitant methotrexate: a randomised phase III trial. ATTRACT Study Group. Lancet 1999;354:19321939.

18. Tarantino G, Conca P, Basile V, et al. A prospective study of acute drug-induced liver injury in patients suffering from non-alcoholic fatty liver disease. Hepatol Res 2007;37:410-415.

19. Feagins LA, Flores A, Arriens C, et al. Nonalcoholic fatty liver disease: a potential consequence of tumor necrosis factor-inhibitor therapy. Eur J Gastroenterol Hepatol 2015;27:1154-116o.

20. McGowan CE, Jones P, Long MD, Barritt AS 4th. Changing shape of disease: nonalcoholic fatty liver disease in Crohn's disease-a case series and review of the literature. Inflamm Bowel Dis 2012;18:49-54.

21. Sourianarayanane A, Garg G, Smith TH, Butt MI, McCullough AJ, Shen B. Risk factors of non-alcoholic fatty liver disease in patients with inflammatory bowel disease. J Crohns Colitis 2013;7:e279-e285.

22. Sartini A, Leonardi F, Gitto S, Di Girolamo M, Villa E. Letter: TNF $\alpha$ inhibitors and prevalence of fatty liver disease in chronic inflammatory diseases. Aliment Pharmacol Ther 2015;42:489.

23. Amacher DE. Female gender as a susceptibility factor for drug-induced liver injury. Hum Exp Toxicol 2014;33:928939.

24. Chalasani N, Bjornsson E. Risk factors for idiosyncratic drug-induced liver injury. Gastroenterology 2010;138:2246-2259.

25. Soldin OP, Mattison DR. Sex differences in pharmacokinetics and pharmacodynamics. Clin Pharmacokinet 2009;48:143-157.

26. Gonnet-Gracia C, Barnetche T, Richez C, Blanco P, Dehais J, Schaeverbeke T. Anti-nuclear antibodies, anti-DNA and $\mathrm{C}_{4}$ complement evolution in rheumatoid arthritis and ankylosing spondylitis treated with TNF-alpha blockers. Clin Exp Rheumatol 2008;26:401-407.

27. de Vries HS, van Oijen MG, Driessen RJ, et al. Appropriate infliximab infusion dosage and monitoring: results of a panel meeting of rheumatologists, dermatologists and gastroenterologists. Br J Clin Pharmacol 2011;71:7-19.

28. Garcia Aparicio AM, Rey JR, Sanz AH, Alvarez JS. Successful treatment with etanercept in a patient with hepatotoxicity closely related to infliximab. Clin Rheumatol 2007;26:811-813.

29. Massarotti M, Marasini B. Successful treatment with etanercept of a patient with psoriatic arthritis after adalimumab-related hepatotoxicity. Int J Immunopathol Pharmacol 2009;22:547-549.

30. Cravo M, Silva R, Serrano M. Autoimmune hepatitis induced by infliximab in a patient with Crohn's disease with no relapse after switching to adalimumab. BioDrugs 2010;24 Suppl 1:25-27.

31. Kooloos WM, de Jong DJ, Huizinga TW, Guchelaar HJ. Potential role of pharmacogenetics in anti-TNF treatment of rheumatoid arthritis and Crohn's disease. Drug Discov Today 2007;12:125-131.

32. Diehl AM. Cytokine regulation of liver injury and repair. Immunol Rev 2000;174:160-171. 